\title{
Nutritional recovery in HIV-infected and HIV- uninfected children with severe acute malnutrition
}

\author{
P Fergusson, ${ }^{1}, 2$ J Chinkhumba, ${ }^{3}$ C Grijalva-Eternod, ${ }^{4}$ T Banda, ${ }^{5} \mathrm{C}$ Mkangama, ${ }^{5}$ \\ A Tomkins ${ }^{4}$
}

${ }^{1}$ Liverpool School of Tropical Medicine, Liverpool, UK; ${ }^{2}$ Action Against Hunger UK, London, UK; ${ }^{3}$ Action Against Hunger Malawi, Lilongwe, Malawi; ${ }^{4}$ Centre for International Health and Development, Institute of Child Health, London, UK: ${ }^{5}$ Ministry of Health, Lilongwe, Malawi

Correspondence to: Pamela Fergusson, Liverpool School of Tropical Medicine, Disease Control Group, Pembroke Place, Liverpool L3 50A, UK; pamela.fergusson@ gmail.com

Accepted 13 October 2008 Published Online First

31 October 2008

\section{ABSTRACT}

Objective: Few studies have reported on nutritional recovery, survival and growth among severely malnourished children with HIV. This study explores nutritional recovery in HIV-infected and HIV-uninfected children during inpatient nutrition rehabilitation and 4 months of follow-up.

Design: Prospective cohort study.

Setting: Lilongwe district, Malawi.

Main outcome measures: Weight gain, anthropometrics.

Results: In our sample of 454 children with severe acute malnutrition (SAM), $17.4 \%(n=79)$ of children were HIV infected. None of the children were on antiretroviral therapy upon admission. Among the HIV-infected children, $35.4 \%(28 / 79)$ died, compared with $10.4 \%(39 / 375)$ in HIV-uninfected children $(p<0.001)$. All children who survived achieved nutritional recovery $(>85 \%$ weight for height and no oedema), regardless of HIV status. HIVinfected children had similar weight gain to HIV-uninfected children (8.9 vs $8.0 \mathrm{~g} / \mathrm{kg} / \mathrm{d}$, not significant (NS)). Mean increases in z-scores for both subscapular (2.72 vs 2.69, NS) and triceps (1.26 vs 1.48, NS) skinfolds were similar between HIV-infected and HIV-uninfected children, respectively, during nutrition rehabilitation. 362 children were followed for 4 months, at which time mean weight for height z-score was similar in HIV-infected and HIVuninfected children $(-0.85$ vs -0.64 , NS).

Conclusions: HIV-infected children with SAM have higher mortality rates than HIV-uninfected children. Among those who survive, however, nutritional recovery is similar in HIV-infected and HIV-uninfected children.

Case fatality rates among children with severe acute malnutrition (SAM) are often as high as 20-30\%, ${ }^{1-4}$ particularly among children with SAM complicated by infection. HIV infection is common in children with SAM in sub-Saharan Africa, particularly in urban referral hospitals. ${ }^{13-10}$ Severely malnourished children with HIV infection have an increased risk of mortality compared with their HIV-uninfected counterparts (relative risk $=3.41,95 \%$ CI 2.24 to 5.20$){ }^{4}$

Few studies, however, have reported on nutritional recovery, survival and growth among severely malnourished children with HIV. Two studies in Malawi, of children with SAM treated as outpatients with ready to use therapeutic food, reported nutritional recovery. In the first study, nutritional recovery, as defined by achieving a weight for height $\mathrm{z}$-score (WHZ) $>-0.5$, was $78 \%$ (202/260) among the children overall and 59\% (46/78) among HIV-infected children. ${ }^{9}$ In the second study, 56\% (52/93) of HIV-infected

\section{What is already known on this topic}

Among children with severe acute malnutrition, HIV-infected children are more likely to die than HIV-uninfected children; at particular risk of mortality are those HIV-infected children with advanced disease and low CD4\%.

\section{What this study adds}

HIV-infected children with severe acute malnutrition who survive nutritional rehabilitation achieve and maintain nutritional recovery as well as HIVuninfected children.

children achieved nutritional recovery, defined as $100 \%$ weight for height index $(\mathrm{W} / \mathrm{H}) .{ }^{8}$ Sandige et al (2004) reported a significantly slower weight gain in HIV-infected children compared with HIVuninfected children (mean (SD) $3.6(4.7) \mathrm{g} / \mathrm{kg} / \mathrm{d}$ vs $5.6(4.0) \mathrm{g} / \mathrm{kg} / \mathrm{d}, \mathrm{p}<0.001)$. These two studies however, do not give a complete picture of nutritional recovery in HIV, as children were recruited after a period of hospital stabilisation. Mortality was not described during this period. ${ }^{89}$

In view of the limited data from long-term community follow-up of children with SAM and HIV after discharge, we measured nutritional recovery in HIV-infected and HIV-uninfected children during inpatient rehabilitation and after 4 months of follow-up.

\section{METHODS}

We carried out a prospective study, measuring survival and nutritional recovery during nutritional rehabilitation, on a cohort of HIV-infected and HIV-uninfected children with SAM. The study was carried out at three nutritional rehabilitation units (NRUs) in Lilongwe District, central Malawi; two belonging to the Ministry of Health $(\mathrm{MOH})$ and one to the Christian Health Association of Malawi (CHAM). Kamuzu Central Hospital NRU is situated in Lilongwe, capital city of Malawi, and is a 1000-bed referral hospital for Malawi's central region. The other two NRUs are situated in rural areas; one, with 200 beds, at St Gabriel's mission hospital; the other, with 100 beds, at Mitundu Community Hospital.

All children aged between 6 and 60 months, with either a $\mathrm{W} / \mathrm{H}$ of less than $70 \%,{ }^{11}$ the presence 
of bilateral pitting oedema or with a mid-upper arm circumference of $<11 \mathrm{~cm}$, were eligible for recruitment. ${ }^{12}$ Written consent to join the study and to test the child for HIV was obtained from all guardians who agreed to have their children join the study (95\% of guardians were mothers). Testing usually occurred within the first 3 days of admission to the NRU. All children, for whom consent to join the study was obtained, had blood collected for HIV and CD4 testing.

Severely malnourished children were recruited at the outpatients department and referred for therapeutic feeding in the NRU. ${ }^{12}$ A small proportion of children with SAM had serious medical complications; they were referred directly to the paediatric ward for medical and nutritional care, and were not recruited into the study. Children who had been initially admitted to the paediatric ward but subsequently transferred to the NRU were recruited to the study. Five point five per cent (25/454) of children who were admitted to the NRU, and became part of our study but who subsequently developed serious medical complications were transferred to the paediatric ward and were lost to follow-up.

Nutritional rehabilitation, using F75 and F100, of all severely malnourished children, was carried out in accordance with the Malawi MOH Guidelines for the Management of Severe Acute Malnutrition $^{13}$; adapted from the World Health Organization (WHO) Guidelines for the Inpatient Treatment of Severely Malnourished Children. ${ }^{12}$ All children received an equal standard of care regardless of their guardian's consent or refusal to join the study.

Nutritional rehabilitation is carried out in three phases. ${ }^{12} 13$ Children were weighed on admission and daily during phase I and transitional phase, then on alternate days during phase II using a paediatric Salter scale with an accuracy of $100 \mathrm{~g}$. Height measurements were taken on admission and discharge from the NRU using a paediatric height board with an accuracy of $1 \mathrm{~mm}$. Weight change in oedematous children was measured from lowest weight to discharge weight and in marasmic children from admission weight to discharge weight. Skinfold thickness measurements were taken at two sites as a proxy for body fat. Subscapular and triceps skinfold thickness was measured using a Holtain Tanner/Whitehouse Caliper (Holtain Ltd, Crosswell, UK), on admission and repeated on discharge from the NRU, with an accuracy of $1 \mathrm{~mm}$. Three readings for each skinfold measurement were taken each time and the mean of the three readings was used for calculations.

Children were discharged from the NRU back into the community upon reaching nutritional recovery $(\mathrm{W} / \mathrm{H}$ of $85 \%$ and no presence of oedema for 10 days). Their nutritional rehabilitation and monitoring continued at the supplementary feeding clinics for 4 months, where they were seen every fortnight to receive food rations $(5 \mathrm{~kg}$ corn soya blend mixed with $500 \mathrm{ml}$ of cooking oil) together with routine nutritional and medical assessment. Children were visited at home for follow-up if they failed to attend the supplementary feeding clinics. Urban and rural households were defined by local community-based health staff using addresses, maps and local knowledge.

Cotrimoxazole was prescribed in Malawi during this period for prophylaxis and treatment of known HIV-exposed and HIVinfected children, although there were some interruptions in supply. Paediatric antiretroviral therapy (ART) was not available in Malawi when the study commenced, and none of the children were on ART upon study admission. All mothers were offered voluntary counselling and testing (VCT); however, entry into the study required them to accept an anonymous, linked HIV test for themselves and their child. In May of 2006, with the advent of paediatric ART availability, the study testing protocol was changed to require new study participants to accept HIV testing. HIV-infected mothers and children were referred for HIV treatment and care under $\mathrm{MOH}$ guidelines. Twelve HIV-infected children were recruited after this change in guidelines. None of these children were on ART upon NRU discharge. It is unknown how many of these children were initiated in ART under $\mathrm{MOH}$ care after discharge from the NRU. All children were treated for SAM regardless of the uptake of VCT by their guardians.

HIV was diagnosed in children aged $>18$ months using two rapid tests: Abbott Determine HIV 1 and 2 (Abbott Laboratories, Abbott Park, Illinois, USA) and Trinity UNIGOLD HIV 1 Rapid (Trinity Biotech, Bray, UK). 'Positive' and 'negative' results were those with concordant results for both tests. Discordant results were confirmed using a Bio-Rad Western Blot. HIV diagnosis for children aged $<18$ months was carried out once using DNA polymerase chain reaction, using Roche Amplicor HIV-1 DNA assay (version 1.5) (Roche Diagnostics, Pleasanton, California, USA). CD4 count (expressed as percentage of total lymphocytes) was measured using a Beckman Coulter EPICS II machine (Beckman Coulter, Fullerton, California, USA). HIV and CD4 testing was carried out by the University of North Carolina Laboratories in Lilongwe, Malawi. ${ }^{14}$

The study was carried out from May 2005 to November 2006, recruiting a total of 454 children. All data were double entered in Malawi, and checked for consistency. Ethical approval was given by the National Health Sciences Research Committee of Malawi.

\section{STATISTICAL ANALYSIS}

Weight and height values were transformed to weight for age $\mathrm{z}$ score (WAZ) and weight for height z-score (WHZ) using EpiInfo (version 3.4.3), based on the WHO/Centre for Disease Control National Center for Health Statistics growth references. ${ }^{11}$ Skinfold measurements were transformed into z-scores, based on the new WHO growth references (WHO, 2007), using the $1 \mathrm{~ms}$ Growth programme (version 2.4).

Of a sample of 454 children, 14 children had missing birth date data, meaning WAZ could not be calculated for 14 children and skinfold data could not be calculated for 12 children. Records for presence of oedema were missing for two children. Additionally, 91 children had no skinfold measurements at admission and were excluded from the initial comparisons. Furthermore, no skinfold data were available at discharge for another 51, and a further six children who died or were transferred to the paediatric ward, leaving a final sample of 258 children for comparing the change of skinfold data between admission and discharge.

Data analysis was carried out using SPSS version 14.0. Comparisons between groups were analysed using Chi-square and between means using the Mann-Whitney $U$ test.

\section{RESULTS}

There were 621 eligible children admitted to the NRUs. Eighteen point five per cent $(115 / 621)$ of carers declined. In $10 \%(52 / 506)$ of children HIV diagnosis was not made, either because they died before testing $(22 / 52)$ or had clotted blood samples (30/52). Thus, there were 454 children with known HIV results. 
Table 1 Anthropometrics by HIV status on admission to the study

\begin{tabular}{|c|c|c|c|c|c|c|c|}
\hline & \multicolumn{3}{|c|}{ HIV positive } & \multicolumn{3}{|c|}{ HIV negative } & \multirow{2}{*}{$\begin{array}{l}\text { Difference } \\
\text { p Value }\end{array}$} \\
\hline & No & Mean & SD & No & Mean & SD & \\
\hline Age (months) & 79 & 21.6 & 10.5 & 375 & 23.6 & 10.0 & 0.052 \\
\hline Weight $(\mathrm{kg})$ & 79 & 6.56 & 1.70 & 374 & 7.45 & 1.94 & $<0.001$ \\
\hline Oedema-free weight $(\mathrm{kg})^{*}$ & 70 & 6.27 & 1.48 & 361 & 7.36 & 1.77 & $<0.001$ \\
\hline WAZ admission & 74 & -4.14 & 1.0 & 365 & -3.74 & 1.25 & 0.011 \\
\hline WHZ admission & 79 & -2.74 & 1.11 & 374 & -2.29 & 1.42 & 0.007 \\
\hline MUAC $\dagger$ & 16 & 11.4 & 1.75 & 148 & 12.6 & 1.65 & 0.001 \\
\hline Triceps skinfold z-score & 54 & -3.10 & 2.36 & 297 & -2.32 & 2.10 & 0.018 \\
\hline Subscapular skinfold z-score & 54 & -4.56 & 3.63 & 297 & -3.98 & 2.77 & 0.3 \\
\hline
\end{tabular}

*0edema-free weight is defined as the lowest weight during admission.

$\dagger$ On admission MUAC was only taken for children who were $>75 \mathrm{~cm}$ tall.

MUAC, mid upper arm circumference; WAZ, weight for age z-score; WHZ, weight for height z-score.

In our sample of 454 children with SAM, 17.4\% $(n=79)$ of children were HIV infected. Among the HIV-infected children, $35.4 \%(28 / 79)$ died during nutritional rehabilitation, compared with $10.4 \%(39 / 375)$ in HIV-uninfected children $(p<0.001)$. All children who survived achieved nutritional recovery, regardless of HIV status (fig 2).

HIV-infected children weighed less on admission and had a lower oedema-free weight (table 1) (both $\mathrm{p}<0.01$ ). Overall, $86.7 \%(392 / 452)$ of children were oedematous, and fewer HIVinfected children had oedema than HIV-uninfected children (56/79, $70.9 \%$ vs 336/373, 90.1\%, p=0.01). HIV-infected children had significantly lower values of WHZ and WAZ (both $p<0.01)$, and lower triceps skinfold z-scores $(p=0.018)$. Subscapular skinfold z-score values were lower for HIV-infected children but this difference was not statistically significant.

Oedematous children gained weight significantly less quickly $(7.9 \mathrm{~g} / \mathrm{kg} / \mathrm{d}, \mathrm{n}=315)$ than marasmic children $(10.7 \mathrm{~g} / \mathrm{kg} / \mathrm{d}$, $\mathrm{n}=45)(\mathrm{p}=0.001)$. There was no significant difference in rate of weight gain for marasmic HIV-infected $(10 \mathrm{~g} / \mathrm{kg} / \mathrm{d}, \mathrm{n}=14)$ or marasmic HIV-uninfected children $(11.1 \mathrm{~g} / \mathrm{kg} / \mathrm{d}, \mathrm{n}=28)$. HIVinfected and HIV-uninfected children with oedema also had similar rates of weight gain $(8.6 \mathrm{~g} / \mathrm{kg} / \mathrm{d}, \mathrm{n}=28 \mathrm{vs} 7.7 \mathrm{~g} / \mathrm{kg} / \mathrm{d}$, $\mathrm{n}=284$, not significant (NS)).

HIV-infected children gained more weight than HIV-uninfected children (1.45 kg vs $1.21 \mathrm{~kg} ; \mathrm{p}=0.03$ ). Although HIVinfected children had a longer stay in the NRU (28.8 days vs 24.3 days; $p=0.03)$, their rate of weight gain was slightly higher $(8.9 \mathrm{~g} / \mathrm{kg} / \mathrm{d}$ vs $8.0 \mathrm{~g} / \mathrm{kg} / \mathrm{d}$, NS). CD4 count data were available for $65 \mathrm{HIV}$-infected children; 45/65 children had a CD $4 \%$ of $<20 \%$. HIV-infected children with a CD $4 \%<20 \%$ gained weight less rapidly than those with a CD4\% $>20 \%$ (7.6 g/kg/d vs $11.9 \mathrm{~g} / \mathrm{kg} / \mathrm{d}, \mathrm{NS})$.

Using the 2006 WHO recommendations for starting paediatric ART according to CD4\% by child age group, ${ }^{15}$ ART is recommended for children: $\leqslant 11$ months of age with CD4\% $<25 \%$; 12-35 months with CD4\% <20\%; and 36-59 months with $\mathrm{CD} 4 \%<15 \%$. Sixty-nine point two per cent $(45 / 65)$ of

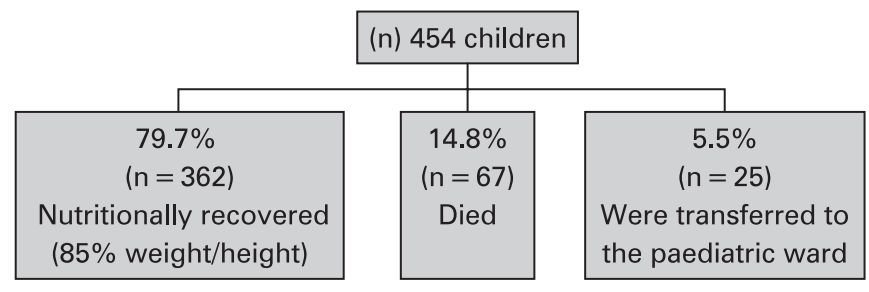

Figure 1 Outcome of nutritional rehabilitation.
HIV-infected children with SAM required ART (7/9 $\leqslant 11$ months, 32/48 12-35 months and 6/8 36-59 months).

HIV-infected children who required ART by WHO criteria had a mean weight gain of $7.6 \mathrm{~g} / \mathrm{kg} / \mathrm{d}$ (SD 4.4) while those who did not yet qualify for ART gained $11 \mathrm{~g} / \mathrm{kg} / \mathrm{d}$ (SD 6.1) (NS).

Skinfold data of children who recovered nutritionally are shown in fig 3. Subscapular skinfold z-scores were similar on admission and discharge between HIV-infected and HIVuninfected children. HIV-infected children, however, had significantly lower triceps skinfolds than HIV-uninfected children on admission. Despite this initial difference, the mean change in z-score values for both subscapular and triceps skinfolds was similar for HIV-infected and HIV-uninfected children.

Eight point five per cent (34/398) of children were lost to follow-up after discharge from the NRU (12 HIV infected, 22 HIV uninfected). Three hundred and sixty-four children were followed for 4 months following discharge. Eleven children died during the 4 months of follow-up after discharge from the NRU (four HIV infected, seven HIV uninfected). At 4 months of follow-up, mean WHZ was similar in both HIV-infected and HIV-uninfected children ( -0.85 vs -0.64 , NS). From admission

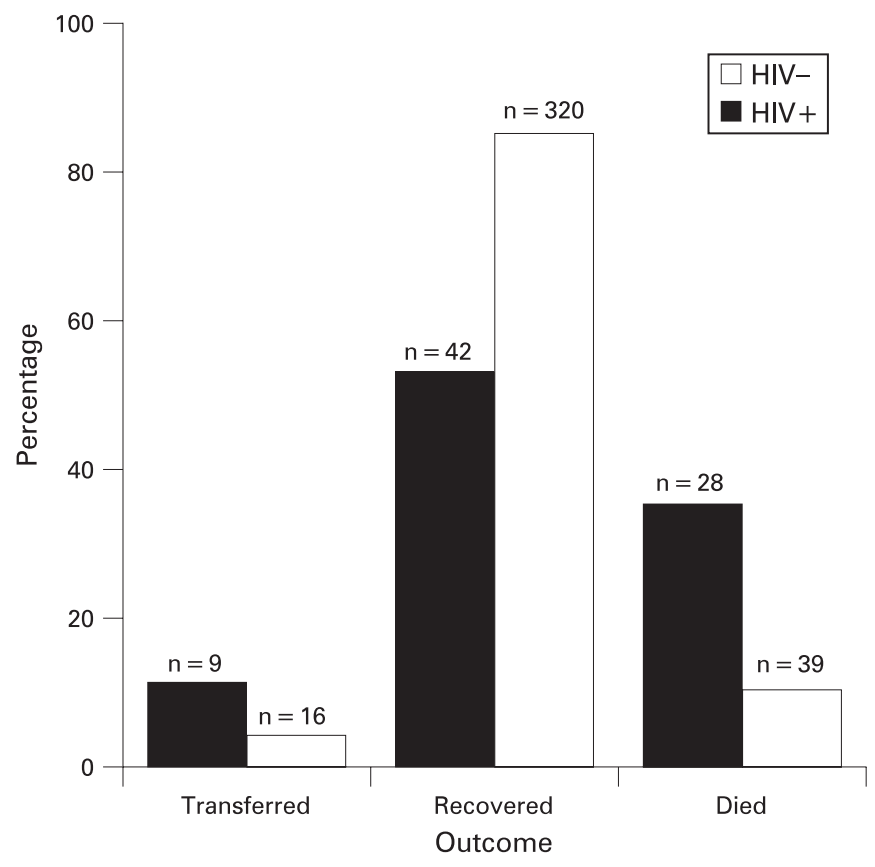

Figure 2 Outcome of nutritional rehabilitation within the nutritional rehabilitation unit. 
Figure 3 Outcome of nutrition rehabilitation in HIV-infected and HIVuninfected children within the nutritional rehabilitation unit.

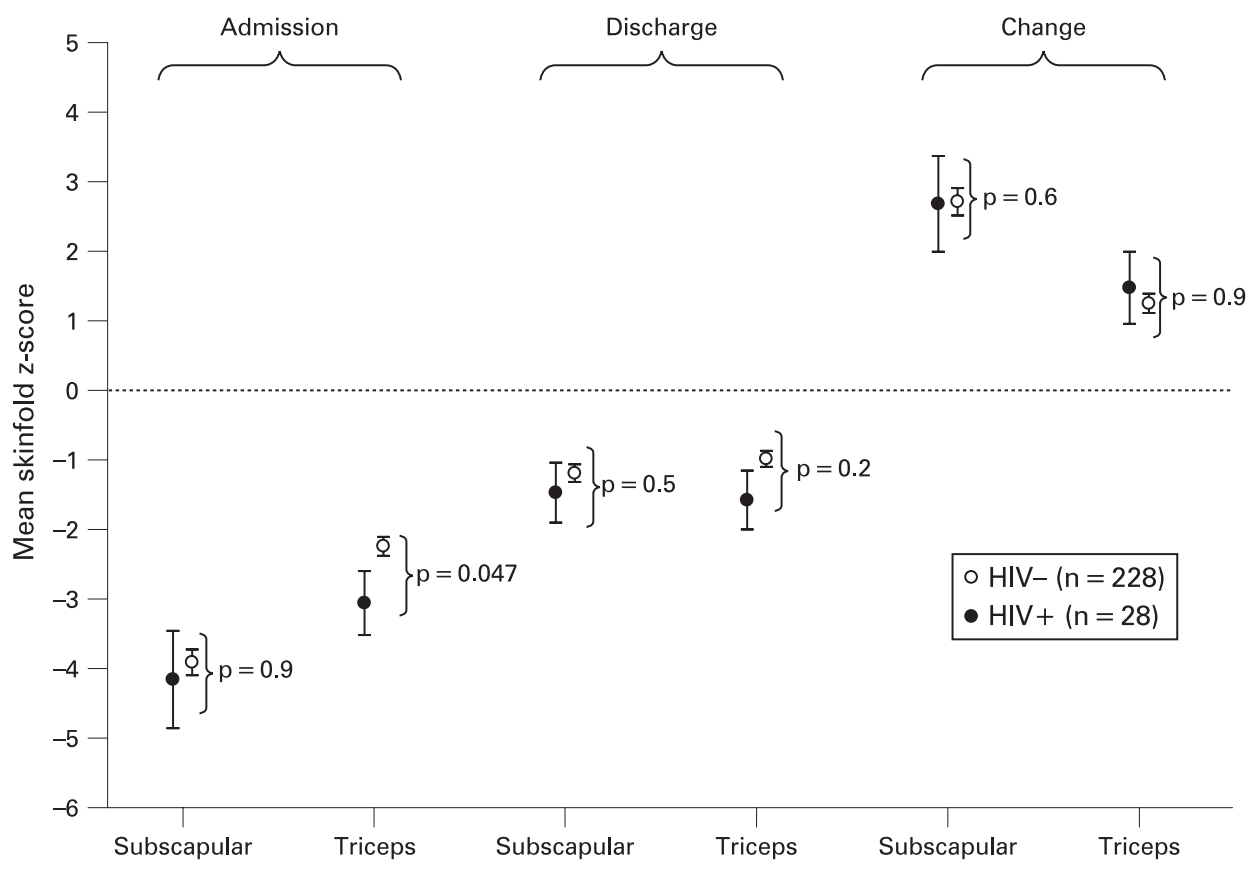

to 4 months of follow-up, WHZ increased by 1.94 z-scores (SD 1.75) in HIV-infected children and by $1.64 \mathrm{z}$-scores (SD 1.84) in HIV-uninfected children (NS). Furthermore, mid upper arm circumference at follow-up was similar between HIV-infected children $(13.7 \mathrm{~cm}, \mathrm{SD} 1.23)$ and HIV-uninfected children (13.9 cm SD 1.39) (NS). Data on appetite during community follow-up were available for 344 children; of these, $98.1 \%$ had good appetites $(n=341)$. Additionally, only three children $(0.9 \%)$ had relapsed and required re-admission.

\section{DISCUSSION}

Nutritional recovery was achieved in $79.7 \%$ (362/454) of severely malnourished children overall, meeting international minimum standards for recovery from nutritional rehabilitation $(>75 \%) .{ }^{16}$ However, this study shows clearly that HIV-infected children were less likely to achieve nutritional recovery than HIV-uninfected children overall; this is attributable to their higher mortality. Nutritional recovery among the survivors was similarly good among HIV-infected and HIV-uninfected children. Anthropometric measurements for weight, weight for height and skinfold data showed that HIV-infected children were more malnourished than HIV-uninfected children on admission, and were significantly less likely to survive.

Among those children who survive, however, nutritional recovery and growth are similar for HIV-infected and HIVuninfected children. These gains were maintained over 4 months of follow-up post-discharge from the NRU. Also, among children who survived, HIV-infected and HIV-uninfected children experienced similar improvements in triceps and subscapular skinfolds.

We believe this to be an important finding, as it dispels the common belief among many health workers that HIV-infected children with SAM do not recover well. This research shows that investing resources into nutritional rehabilitation can yield benefits in child survival and growth. Furthermore, it is likely that, with improved access to paediatric ART in sub-Saharan Africa, nutritional recovery among HIV-infected children with
SAM will result in improvements in survival, nutritional recovery and growth. ${ }^{17}$

In an earlier publication from the same cohort of children, we reported that mortality was significantly higher in children with lower CD4\%. ${ }^{4}$ The present data show that surviving children with low CD4\%, and those who are eligible for ART according to WHO 2006 guidelines, gained weight more slowly — most likely the result of opportunistic infections and anorexia. It is noteworthy that even among HIV-infected children with SAM who achieved nutritional recovery, CD4\% was low. This underscores the importance of providing access to HIV and CD4\% testing as well as ART treatment for severely malnourished children. In the absence of CD4\% monitoring, however, these data support using severe malnutrition as a clinical criterion for paediatric ART initiation. ${ }^{16}$

Although ART may impact positively on nutritional recovery for some HIV-infected children it is likely that some of the children presenting with low CD4\% and SAM complicated by opportunistic infections will present with advanced disease, and ART may be too late to impact on survival. Incorporating HIV testing and treatment into growth monitoring and into programmes for treatment of moderate malnutrition may help to detect HIV infection at risk at an earlier stage, and improve outcomes.

\section{CONCLUSION}

HIV-infected children with SAM have higher mortality rates than HIV-uninfected children. Among those who survive, however, nutritional recovery is similar in HIV-infected and HIV-uninfected children.

Our findings show that HIV-infected children can achieve and maintain nutritional recovery. However, the majority of those HIV-infected children recovering from SAM have a low CD4\% and require ART. Nutrition rehabilitation programmes are an important entry point for HIV care, and in order to achieve further gains in child growth and survival among HIVinfected severely malnourished children, it is essential to 
integrate HIV services into programmes for nutritional rehabilitation in high HIV prevalence settings. HIV testing, CD4 monitoring and treatment, including cotrimoxazole and ART, should be universally available to children with SAM.

Acknowledgements: The authors would like to thank the participating families; the National AIDS Commission of Malawi, Unicef Malawi, Action contre la Faim, Paris, and Acción contra el Hambre Madrid for their financial support; and the staff at AAH Malawi, Kamuzu Central Hospital, St Gabriels, and Mitundu Hospitals.

Competing interests: None.

Ethics approval: Ethical approval was given by the National Health Sciences Research Committee of Malawi.

Patient consent: Parental consent obtained.

\section{REFERENCES}

1. Bachou H, Tumwine J, Mwadime R, et al. Risk factors in hospital deaths in severely malnourished children in Kampala, Uganda. BMC Pediatr 2006;6:7.

2. Schofield $\mathbf{C}$, Ashworth A. Why have mortality rates for severe malnutrition remained so high? Bull of the World Health Organ 1996;742:223-9.

3. Kessler L, Daley H, Malenga G, et al. The impact of the human immunodeficiency virus type 1 on the management of severe malnutrition in Malawi. Ann Trop Paediatr 2000:201:50-6.

4. Chinkhumba J, Tomkins A, Banda T, et al. The impact of HIV on mortality during inpatient rehabilitation of severely malnourished children in Malawi. Trans $R$ Soc Trop Med Hyg 2008;102:639-44.

5. Amadi B, Kelly P, Mwiya M, et al. Intestinal and systemic infection, HIV, and mortality in Zambian children with persistent diarrhea and malnutrition. J Pediatr Gastroenterol Nutr 2001;325:550-4.
6. Amadi B, Mwiya M, Chomba E, et al. Improved nutritional recovery on an elemental diet in Zambian children with persistent diarrhoea and malnutrition. J Trop Pediatr 2005;511:5-10.

7. Bachou H, Tylleskär T, Downing $\mathrm{R}$, et al. Severe malnutrition with and without HIV-1 infection in hospitalised children in Kampala, Uganda: differences in clinical features, haematological findings and CD4+ cell counts. Nutr J 2006;5:27.

8. Ndekha MJ, Manary MJ, Ashorn P, et al. Home-based therapy with ready-to-use therapeutic food is of benefit to malnourished, HIV-infected Malawian children. Acta Paediatr 2005:942:222-5

9. Sandige H, Ndekha MJ, Briend A, et al. Home-based treatment of malnourished Malawian children with locally produced or imported ready-to-use food. J Pediatr Gastroenterol Nutr 2004;392:141-6.

10. Thurstans S, Kerac M, Maleta K, et al. HIV prevalence in severely malnourished children admitted to nutrition rehabilitation units in Malawi: geographical \& seasonal variations. A cross-sectional study [abstract]. Toronto: International AIDS Conference, 2006.

11. WHO. Management of severe malnutrition: a manual for physicians and other senior health workers. Geneva: World Health Organization, 1999.

12. WHO. Guidelines for the inpatient treatment of severely malnourished children Geneva, 2003.

13. Malawi. Guidelines for the management of severe acute malnutrition. Government of Malawi, 2003.

14. Fiscus SA, Pilcher CD, Miller WC, et al. Rapid, real-time detection of acute HIV infection in patients in Africa. J Infect Dis 2007;1953:416-24.

15. Sphere. Humanitarian charter and minimum standards in disaster response. Geneva: The Sphere Project, 2004.

16. WHO. Antiretroviral therapy of HIV infection in infants and children in resource-limited settings: towards universal access. Recommendations for a public health approach. Geneva, 2006.

17. Heikens GT, Bunn J, Amadi B, et al. Case management of HIV-infected severely malnourished children: challenges in the area of highest prevalence. Lancet 2008;371:1305-7. 